\title{
Mitral valve-in-valve and valve-in-ring: tips, tricks, and outcomes
}

\author{
Luigi Pirelli ${ }^{1}$, Estee Hong ${ }^{2}$, Robert Steffen ${ }^{3}$, Torsten P. Vahl ${ }^{2}$, Susheel K. Kodali ${ }^{2}$, Vinayak Bapat ${ }^{2}$ \\ ${ }^{1}$ Department of Cardiothoracic Surgery, Northwell Health, Lenox Hill Hospital, New York, NY, USA; ${ }^{2}$ Department of Cardiothoracic Surgery, \\ Columbia University Irvine Medical Center, New York, NY, USA; ${ }^{3}$ Minneapolis Heart Institute, Abbott Northwestern Hospital, Minneapolis, USA \\ Correspondence to: Vinayak Bapat, MD. Department of Cardiothoracic Surgery, Abbott Northwestern Hospital Vinayak N. Bapat, Columbia \\ University Irvine Medical Center, 177 Fort Washington Avenue, MHB 77GN-435, New York, NY 10032, USA. Email: vb2445@cumc.columbia.edu.
}

\begin{abstract}
Transcatheter aortic valve implantation is now a mainstay of treatment in patients with aortic stenosis who are considered intermediate, high and prohibitive risk for surgery. Extended use of this innovative platform in treating other conditions has led to its approval in treating degenerated aortic bioprosthesis. Similarly, use of transcatheter devices in treating degenerated mitral bioprosthesis and failed mitral valve repairs with annuloplasty rings has opened a potential alternative to surgery in these patients. Experience in mitral valvein-valve (MVIV) and valve-in-ring (VIR), while still limited, is on the rise. Although similar in many ways to the aortic VIV, it is different with respect to patient selection, planning and procedural steps. Familiarity with the bioprosthetic properties and dimensions can help an operator choose an appropriate transcatheter device and deploy it in an ideal position. Due to greater variability in construction and properties, mitral rings have led to poorer results compared to mitral valve-in-valve. Understanding the properties of mitral rings is critical and has been simplified by us in a stepwise manner. We also describe steps in patient preparation and procedure, which should help operators in performing this procedure. Certain unique complications, such as left ventricular outflow tract obstruction and risk of embolization, are discussed with tips to address these issues. Once these steps are followed, the procedure can be performed with minimal risk and good outcome.
\end{abstract}

Keywords: Valve; transcatheter valves; mitral; ring; repair; valve-in-valve (VIV)

Submitted Dec 12, 2019. Accepted for publication Nov 24, 2020.

doi: $10.21037 /$ acs-2019-mv-169

View this article at: http://dx.doi.org/10.21037/acs-2019-mv-169

\section{Introduction}

Mitral valve replacement and repair have formed the backbone of mitral surgery in the last few decades. Mitral pathology varies from degenerative and ischemic, which are commonly seen in the western world, to rheumatic heart disease, which is predominantly seen in developing countries (1). Mitral valve repair is preferred over mitral valve replacement, as it has shown to be a better prognostic procedure and also postpones the need for a replacement in relatively younger patients (2). This is important, as we do not have an ideal valve replacement substitute for replacement. In younger patients, mechanical valves are preferred, as they can last lifelong but require anticoagulation, whereas in older patients, a bioprosthesis (pericardial or porcine) valve is preferred to eliminate the need for anticoagulation. Bioprosthetic valves, however, degenerate with time, and durability varies from 10 to 15 years in the mitral position (3). Importantly, younger patients are also observed to favor bioprosthetic valves, which means that more patients will likely need subsequent intervention (4). Until recently, redo-surgery was the only option for these patients, but with the success of transcatheter aortic valve replacement (TAVR) and valvein-valve (VIV) for degenerated aortic bioprosthesis, an alternative of mitral valve-in-valve (MVIV) has emerged (5). While similar in many ways, MVIV has certain specific unique aspects with respect to patient selection, procedure planning and postoperative care. Interestingly, as the number of mitral repairs for various pathologies has increased, the number of failures has also risen. Failure can be due to pathology, complexity of repair and/or technical reasons. One of the key elements of any mitral repair is 
annuloplasty. Annuloplasty is performed to reshape and stabilize the mitral annulus and is achieved by suturing an annuloplasty ring/band to the mitral annulus on the atrial side. Broadly, the annuloplasty can be just posterior, as well posterior and anterior, to achieve this. A variety of mitral rings have been manufactured and implanted in the last two decades. Similar to the concept of MVIV, repair failures have been treated by implanting a TAVR device within a ring, which is referred to as mitral valve-in-ring (MVIR) (6).

However, MVIV and MVIR are more challenging procedures compared to TAVR in native valve and aortic VIV. Although MVIV and MVIR share multiple similarities, MVIR is a much more complex procedure than MVIV. In this article, we describe optimal patient selection for MVIV and MVIR, along with a step-by-step approach to both procedures, and discuss possible complications, outcomes, and tips on postoperative care. Some aspects of planning and procedural steps are similar in both MVIV and MVIR and will be described together, while important differences will be discussed separately in detail to help operators plan for optimal results.

\section{Operative technique}

\section{Procedural planning-MVIV}

\section{Identification of the bioprosthesis (MVIV)}

The first step is to identify the type and size of bioprosthesis. In the mitral position, unlike the aortic position, only stented bioprosthesis are used. Details of the bioprosthesis, such as type and size, can be obtained from the operation note, valve card given to the patient, or from the bioprosthesis manufacturer. If this information is not available, the bioprosthesis can be identified from its fluoroscopic appearance (7). The fluoroscopic appearance of each bioprosthesis is unique and is similar to its aortic counterpart (Figure 1).

\section{True internal diameter}

Once the type of the bioprosthesis is identified, the next step is to determine the "true internal diameter (ID)" (8). Manufacturer labeling provides label size, external stent diameter and stent internal diameter. However, these parameters may be misleading when planning a MVIV. True ID is the most important dimension and determines the size of the transcatheter heart valve (THV) to be implanted within a given bioprosthesis. True ID is essentially the mechanical internal diameter after mounting the leaflets on the stent frame (Figure 2) (8). This information can be easily obtained from the MVIV App (Figure 3) (9). While computerized tomography scan (CT) is emerging to be an important imaging modality, CT characterization of different bioprostheses is far from standardized; hence, CT scans can only be used as an adjunct to the sizing information in the MVIV App.

\section{THV type and size}

A THV is selected on the basis of user preference and access route. It is obvious that only THV devices with short profiles can be used in the mitral position (Figure 4). The most experience has been with the SAPIEN balloon expandable platform (SAPIEN, SAPIEN XT and SAPIEN 3, Edwards Lifesciences, Irvine USA). Other THV devices used are INNOVRE, MyVal, Lotus and Direct Flow (10). Size of the THV used is determined by the sizing algorithm of each device. In the MVIV, an at least $2 \mathrm{~mm}$ oversize over the True ID is preferred due to higher closing pressures and risk of embolization. Excessive oversizing may be unfavorable, as it leads to under expansion of the THV device. Under expansion increases the risk of leaflet pin wheeling, possible thrombosis, or decreased durability.

\section{Fluoroscopic appearance}

While important to identify the type of the device, important fluoroscopic landmarks for ideal positioning of the THV device during the procedure must also be identified. An ideal position can be defined as a position where the device is secure and achieves best hemodynamic performance (11). Hence, the neo-annulus of the bioprosthesis, which is the narrowest portion of the bioprosthesis, should be identified. The neo-annulus corresponds to the sewing ring of the bioprosthesis. Some bioprostheses display a sewing ring marker, but in some bioprostheses where the stent frame or stent post markers are visible, identifying the neo-annulus can be tricky (Figure 5). It is then important to know the relationship between the stent frame/stent post markers and the sewing ring to achieve ideal positioning, which is available in the Mitral VIV app.

\section{Procedural planning-MVIR}

Unlike MVIV, MVIR is much more complex due to varying types of rings. It may or may not be easy to identify each ring directly from its fluoroscopic appearance; hence, 

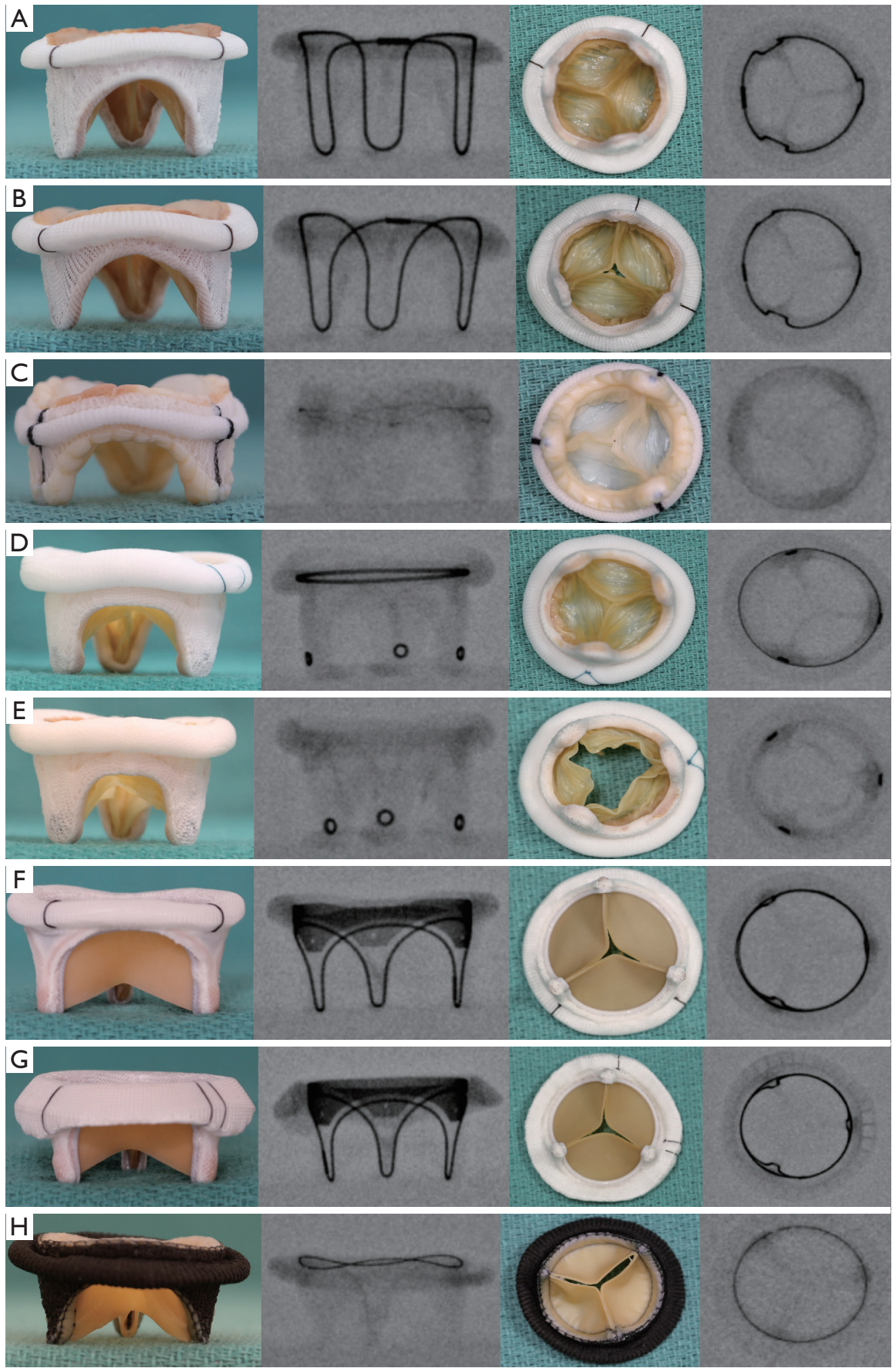

Figure 1 Surgical bioprosthetic mitral valves and their fluoroscopic appearance. (A) Carpentier-Edwards standard porcine; (B) CarpentierEdwards SAV porcine; (C) Epic or Biocor porcine; (D) Hancock II porcine; (E) Mosaic porcine; (F) Perimount Pericardial; (G) Magna Pericardial; (H) Pericarbon More Pericardial. 
A

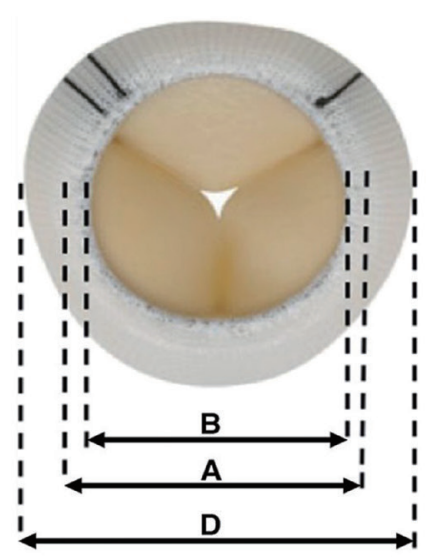

B

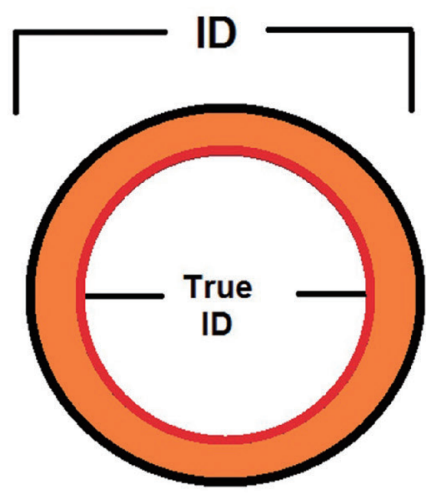

C

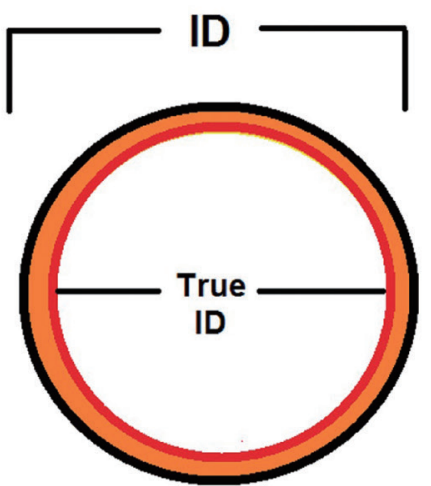

Figure 2 Dimensions of a surgical bioprosthetic valve and true internal diameter (ID). (A) Inflow aspect of the bioprosthesis of a pericardial valve (dimension A: stent frame outer diameter, dimension B: stent frame inner diameter and dimension D: external diameter of sewing ring). (B) Effect of porcine leaflets on ID, stent ID is reduced by at least $2 \mathrm{~mm}$ (true ID porcine valves = stent ID $-2 \mathrm{~mm}$ ). (C) Effect of pericardial leaflets on ID, stent ID is reduced by at least $1 \mathrm{~mm}$ (true ID pericardial valves = stent ID $-1 \mathrm{~mm}$ )

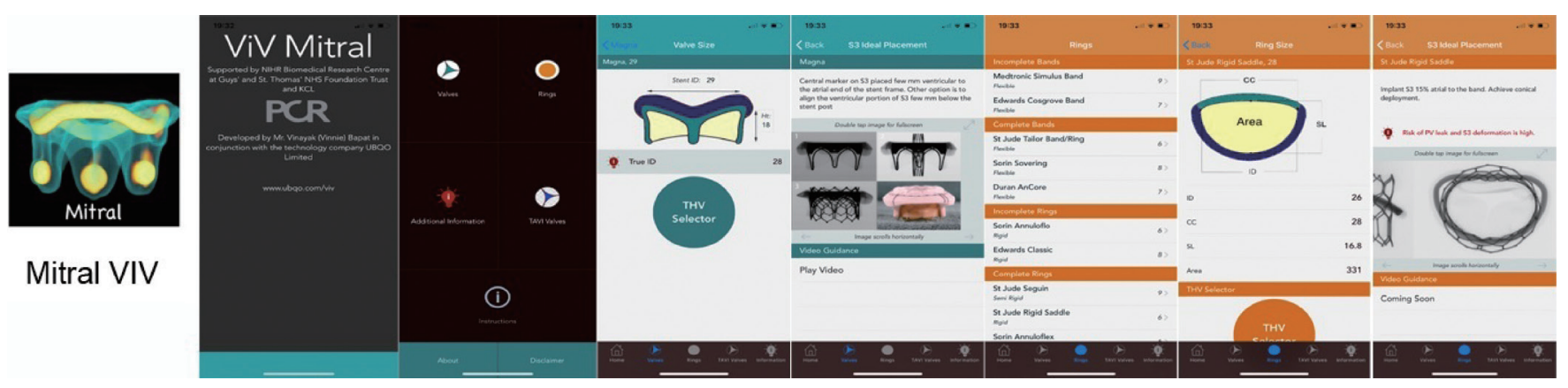

Figure 3 Valve in Valve mitral app: figure demonstrates the App icon and various panels in the workflow. Important information such as valve, ring type, and dimensions (including True ID, size of THV recommended, ideal positioning) are available in the App.

information obtained from the manufacturer and operative note is critical. Some rings, however, are unique in their appearance and can be identified easily (Figure 6). Once identified, it is important to determine suitability of the ring for the MVIR procedure, as, unlike MVIV, not all rings are suitable.

Rings can be classified as: (I) complete, nearly complete or incomplete; (II) rigid, semirigid or flexible. For reason of simplicity, complete and nearly complete will be grouped together as complete.

The four important properties to be considered are: (I) ability to become circular; (II) ability to provide good anchor; (III) size of the ring; (IV) radio-opacity.

\section{Circularity}

Construction and components of the ring can be used to determine achievement of circularity. Incomplete rings/ band obviously cannot become circular due to an absent anterior portion (Figure 7A). A complete ring on the other hand may or may not become circular depending on its components. Rigid rings by nature of their constructions cannot become circular (Figure 7B), semirigid rings can become circular/nearly circular and flexible rings can become circular (Figure 7C). When a ring cannot become circular, the THV is either going to be under expanded or become deformed, resulting in a suboptimal result (Figure $7 B$ ). 
A

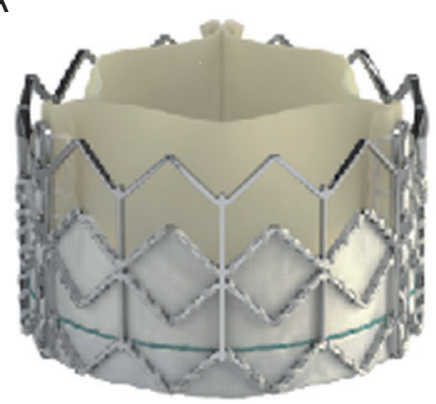

D

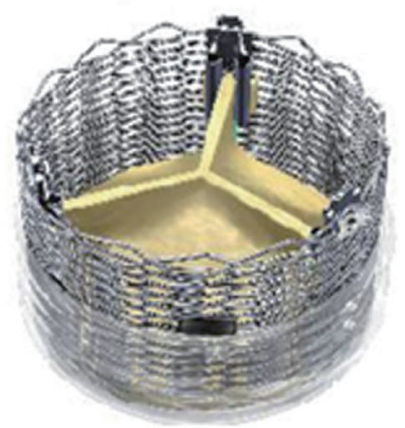

B

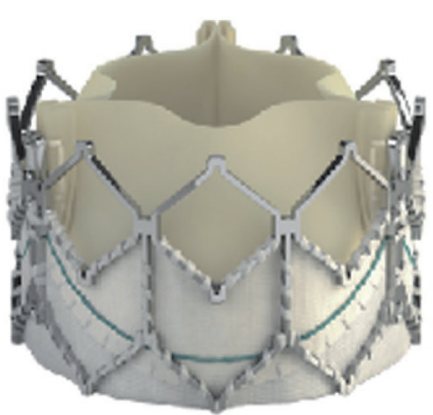

$\mathrm{E}$

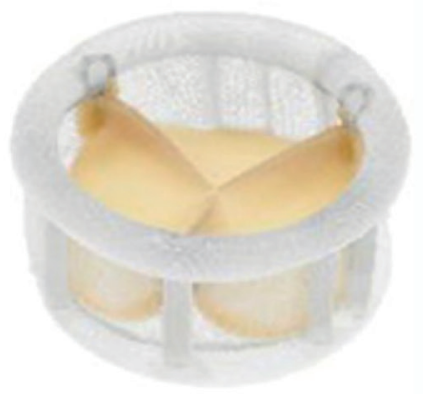

C

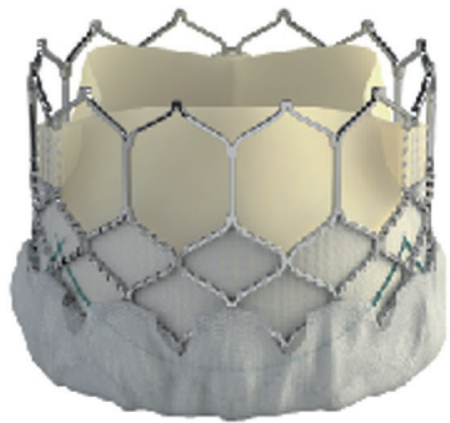

$\mathrm{F}$

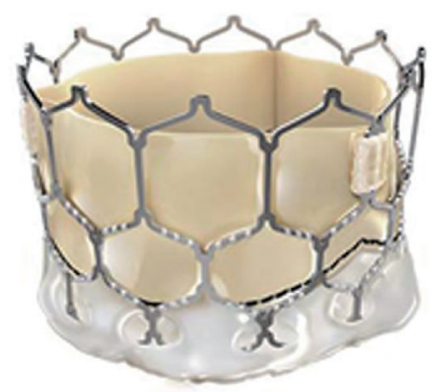

Figure 4 Various transcatheter heart valves (THV) used in mitral position. (A) SAPIEN; (B) SAPIEN XT; (C) SAPIEN 3; (D) Lotus; (E) Directflow; (F) Myval.

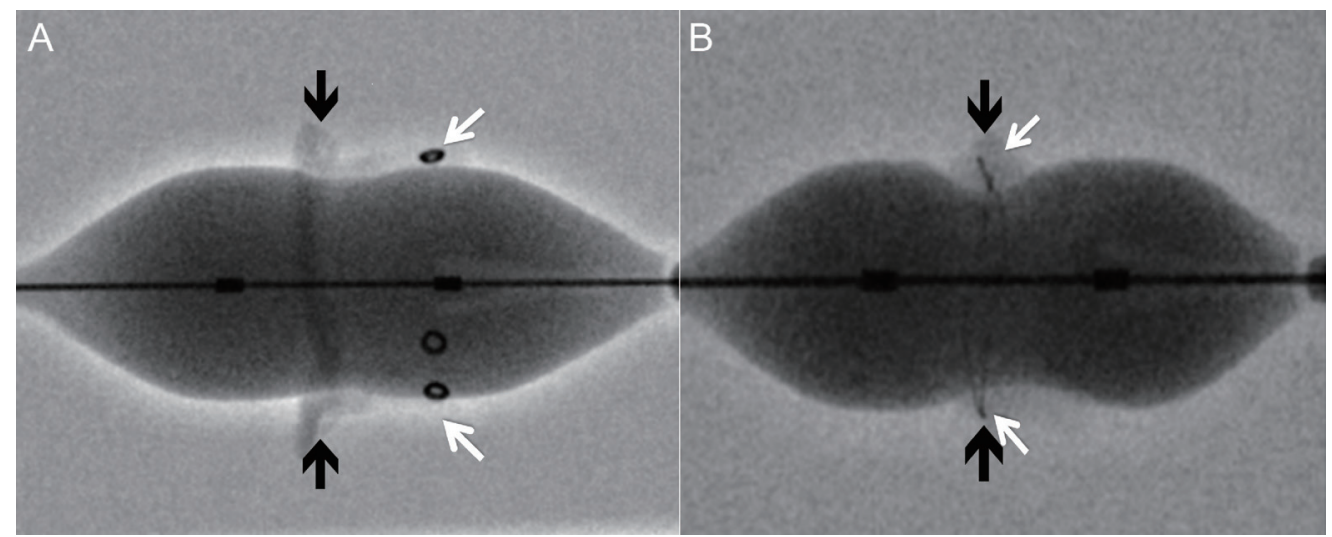

Figure $5 \mathrm{Neo}$-annulus of surgical bioprosthesis and its relationship with fluoroscopic markers. Narrowest portion of the valve is at the sewing ring level. (A) Mosaic mitral valve: the markers are in the stent post (white arrows), which are at different levels compared to the sewing ring level (black arrows). This may make ideal positioning of a transcatheter valve challenging. (B) Epic mitral valve: the markers are in the sewing ring (white arrows) and hence at the same level of the sewing ring (black arrows). This makes ideal positioning of a transcatheter valve easy.

This is very important as poor results can simply be avoided by not performing MVIR in rigid rings. There can be some exceptions, such as rigid rings with a small gap in the anterior portion (Figure 7D), which may develop decent immediate results due to deflection of the gap but will definitely not maintain good mid-term or long-term results. 

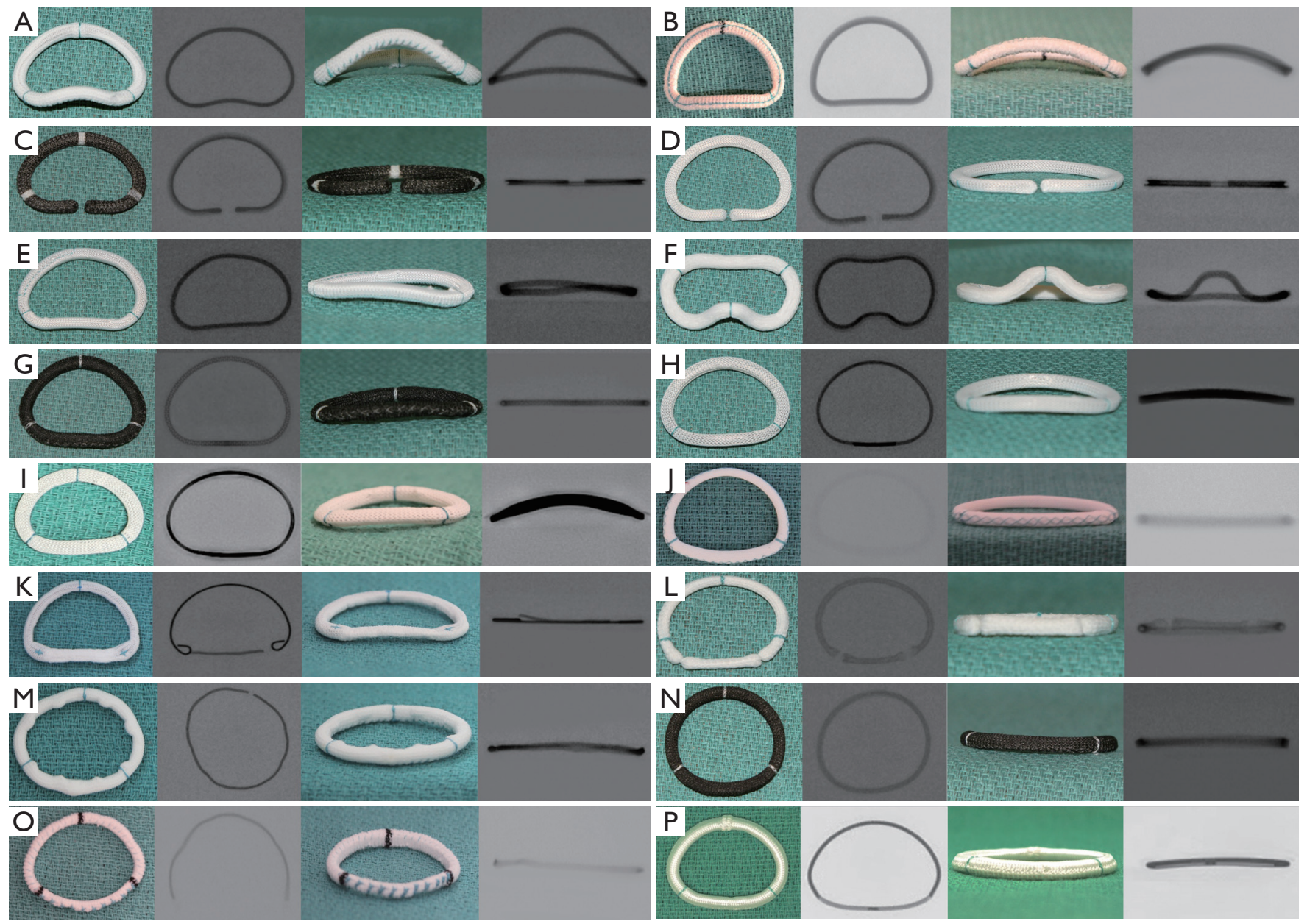

Figure 6 Mitral rings and their fluoroscopic appearance. (A) Profile 3D, Medtronic Inc.; (B) Rigid saddle, Abbott St. Jude; (C) Annuloflo, Livanova; (D) Classic, Edwards Lifesciences; (E) IMR Etiologix, Edwards Lifesciences; (F) Geoform, Edwards Lifesciences; (G) Memo 3D, Livanova; (H) Physio 1, Edwards Lifesciences; (I) Physio 2, Edwards Lifesciences; (J) Seguin, Abbott St. Jude; (K) CG Future, Medtronic Inc.; (L) Annuloflex, Livanova; (M) Duran Ancore, Medtronic Inc.; (N) Sovering, Livanova; (O) Tailor ring/band, Abbott St. Jude; (P) Simulus semirigid ring, Medtronic Inc.

\section{Anchoring}

Although a ring may be able to become circular, it also needs to provide a secure anchor. Without proper anchoring, the THV will embolize. Anchoring is dependent on the rigidity and dimensions. Incomplete rings cannot provide a good anchor. On the other hand, complete rings, depending on their construct, can provide a good anchor. If a ring is too flexible, it can become circular but will not provide a good anchor. Alternatively, a rigid ring can provide a good anchor but does not become circular. Semirigid rings are best of both and provide good anchor, as well as having the ability to become circular or nearly circular. Similarly, flexible rings of certain sizes can also provide good anchor even when stretched, providing enough margin for oversizing.

\section{Size of the ring and dimensions}

The smallest and largest THV available are size 20 and 29, respectively. Hence, choosing the correct size, as well as determining if the ring is too small or large for current THV devices, is important. This is more critical in MVIV and is an area of immense confusion. Rings are usually manufactured from label size 28 to 40, but some are available in smaller or larger sizes. Furthermore, label size definitely does not correspond to any meaningful dimension, and the same label size rings across various types have varying dimensions. Common dimensions provided 
A Incomplete band
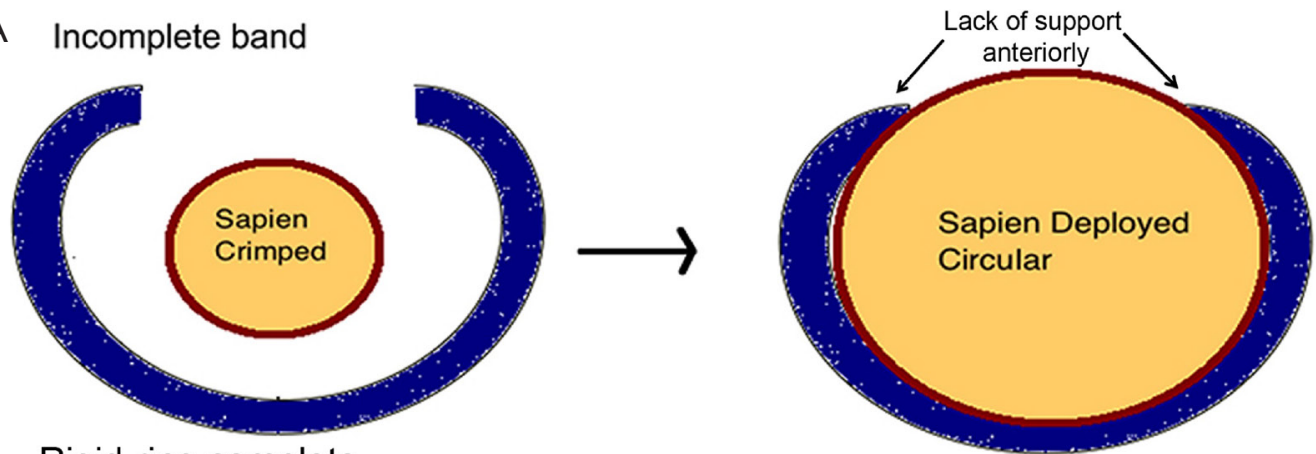

B

Rigid-ring complete
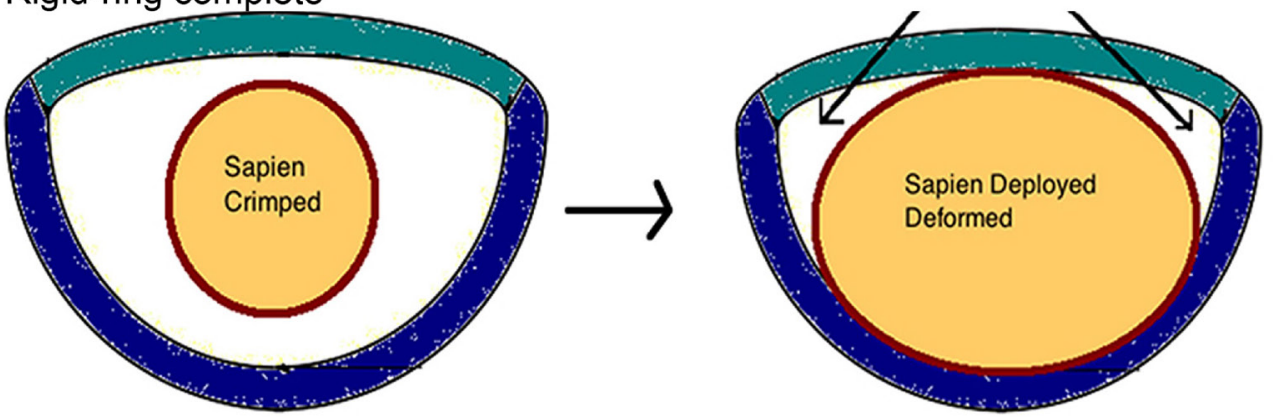

\section{Semi-rigid ring Complete}

C
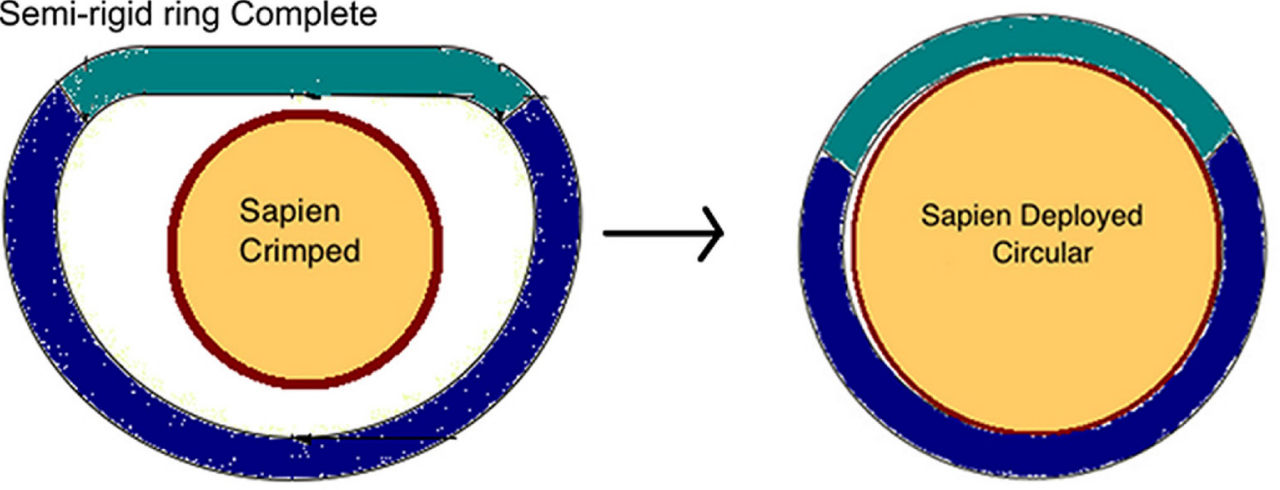

Rigid ring Incomplete
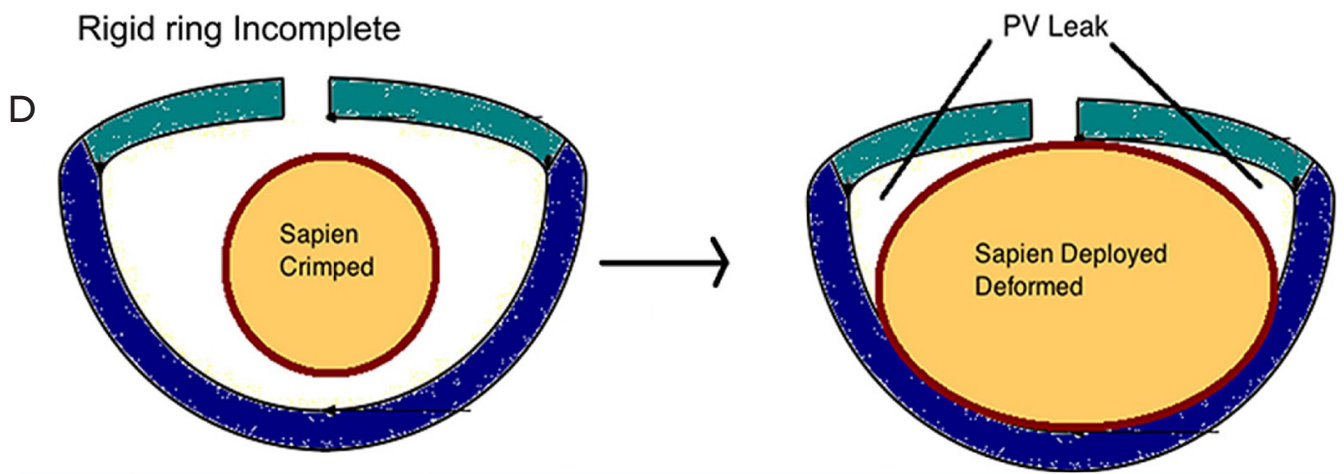

Figure 7 Ability of rings/bands to become circular is critical in influencing success of VIR procedure. (A) Incomplete band does not have anterior support and is hence not suitable. (B) Rigid ring complete cannot become circular and can result in incomplete THV expansion, deformation and leak. (C) Semi-rigid ring complete can become circular or nearly circular and is hence ideal for VIR. (D) Rigid ring complete is very similar to rigid ring complete, but the open ends may allow greater degree of circularity to give acceptable results in some cases. VIR, valve-in-ring. 
by the manufacturers are commissure-to-commissure (CC) length, septal-lateral (SL) length, area, and perimeter. Not all manufacturers provide these dimensions. Additionally, most mitral rings are kidney shaped and become circular or nearly circular after MVIR, resulting in different area and diameter. Therefore, it is important for the user to refer to the MVIV app to determine suitability, as some rings may be too large for a given ring. Usually, any ring larger than label size 34 is not suitable for VIR. With flexible rings, one should also measure the CT dimensions of an imaginary circle and take into consideration the degree of ring extension and malleability to determine suitability.

\section{Radio-opacity}

Rings that are easily seen will make the procedure easier. Rings that are not well seen will need trans-esophageal echo, as well as certain level of experience during the procedure (Figure 67,O). To summarize, when considering a patient for MVIR, determination of suitability should consider the four properties discussed above. Table 1 summarizes the different ring options, their properties, and suitability for a MVIR procedure.

\section{Risk of complications}

\section{Left ventricular outflow tract obstruction}

Left ventricular outflow tract obstruction (LVOTO) is the Achilles heel of any transcatheter mitral intervention. Once a THV is implanted during MVIV or MVIR, the THV pins open the prosthetic or native leaflet to possibly introduce LVOTO (Figure $8 A, B$ ). Furthermore, the THV is now covered partially or fully by these leaflets, and blood flow through the THV stent into the LVOT is not feasible (Figure $8 C, D$ ). Many factors determine risk and degree of LVOTO (Figure 9). The degree of residual LVOT dimensions (neo-LVOT) which is acceptable remains unclear $(12,13)$. The risk for LVOTO is higher after MVIR than MVIV. The risk is more prominent for pericardial valves than porcine and for taller valves than shorter. It is important to stress the implication of this single complication, as LVOTO correlates with poor outcome; therefore, LVOTO prevention is better than attempts to treat post-operational LVOTO. CT analysis to determine neo-LVOTO is critical. Such patients should be reconsidered for surgery. In some patients where the surgical option is not feasible, strategies such as alcohol septal ablation or LAMPOON can be attempted (14).

\section{THV embolization}

One of the unique complications in MVIV and VIR is THV embolization. The systolic pressure creates closing pressures on the mitral valve and is considerably higher than closing pressures observed in the other three valve positions. Reports of immediate, early, and delayed embolization were not uncommon in the early experience of this therapy area (15). Correct oversizing using the True ID hence remains critical. To avoid embolization, it is important to avoid parallel deployment (Figure 10A,B) and to achieve conical deployment (Figure 10C,D).

\section{Access routes}

Three routes have been described for various devices: transapical (TA), trans-septal (TS) and trans-atrial (Ta) (Figure 11). The majority of the early cases were performed by TA approach, but TS is now the predominant approach due to its less invasive nature. Certain THV, however, can only be implanted through the TA approach, such as Lotus and Directflow. The SAPIEN family of THV and MyVal on the other hand can be implanted through either of the approaches. Both TA and TS approaches will be described in detail later.

\section{Preparation}

The procedure should be performed in a hybrid or a well-equipped catheter laboratory with facilities for hemodynamic support and, if needed, open heart surgery for bail out. A clear plan in terms of bail out and equipment needed should be available. Risk of embolization and serious complications such as left ventricular perforation and significant LVOTO are rare but can be life threatening if the bailout plan is unclear.

The procedure should be performed under general anesthesia with transesophageal echocardiography (TEE) guidance. TEE provides excellent imaging guidance during the procedure and can also help rule out or diagnose left atrial thrombus and para-valvular leak if present. In certain cases, such as a mitral ring not visible under fluoroscopy, TEE is the main imaging modality to guide and confirm ideal implant position. TEE can also help to check post implant gradients, valve function and pericardial effusion and assess LVOTO.

Once the TEE probe is inserted and central lines are secured, defibrillation pads are placed. Before preparing the 


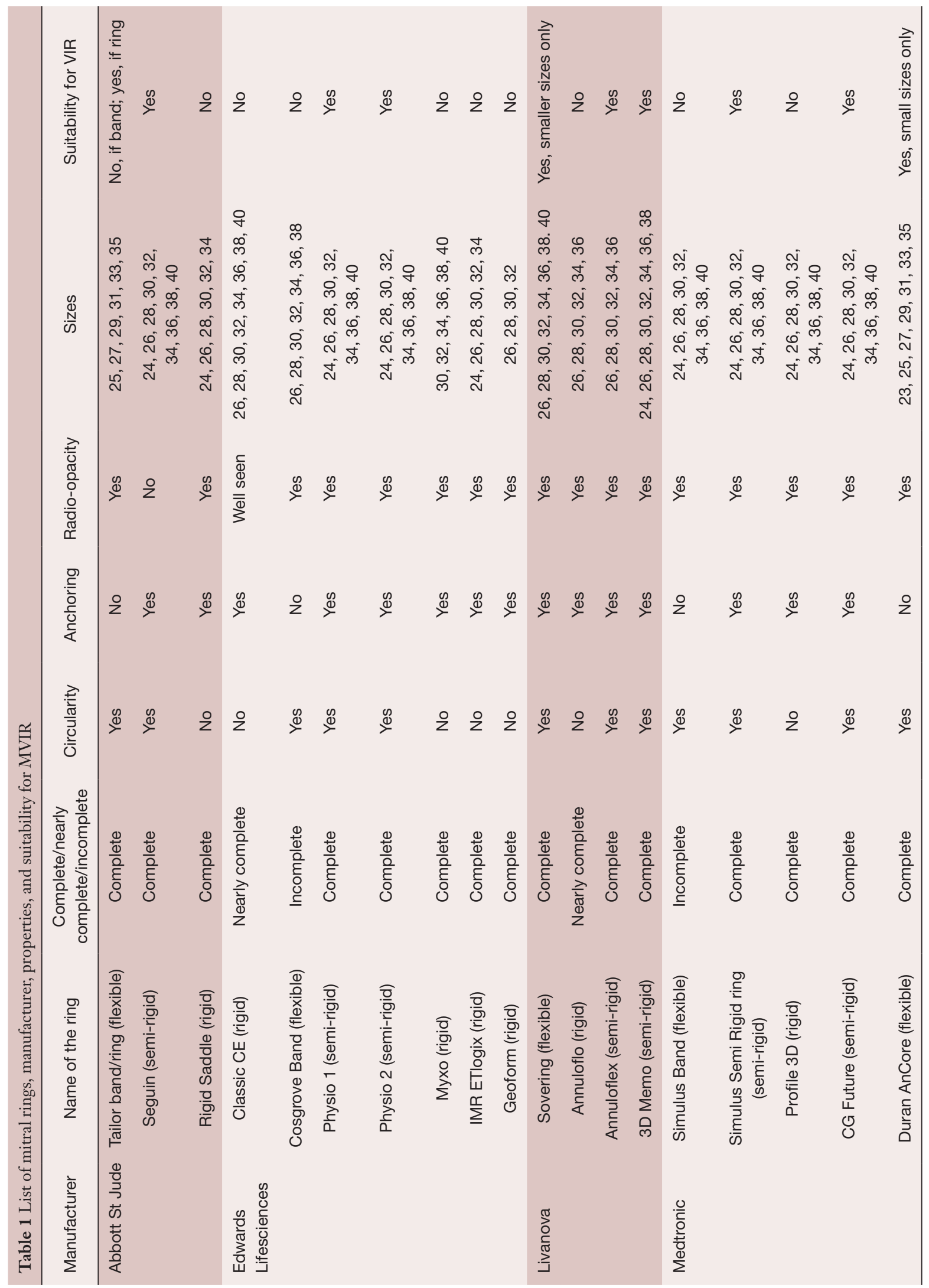


A

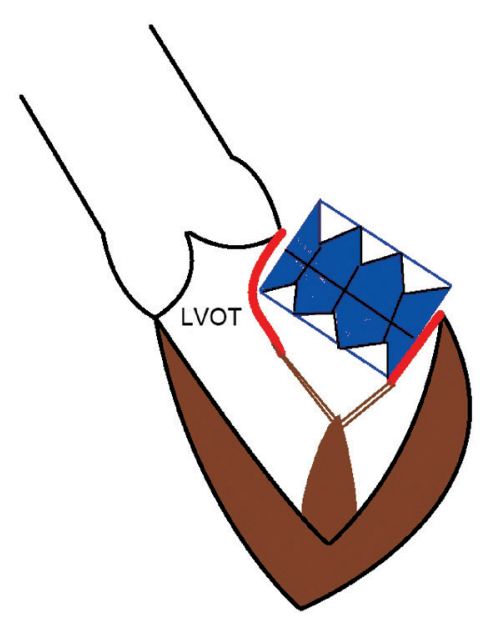

B

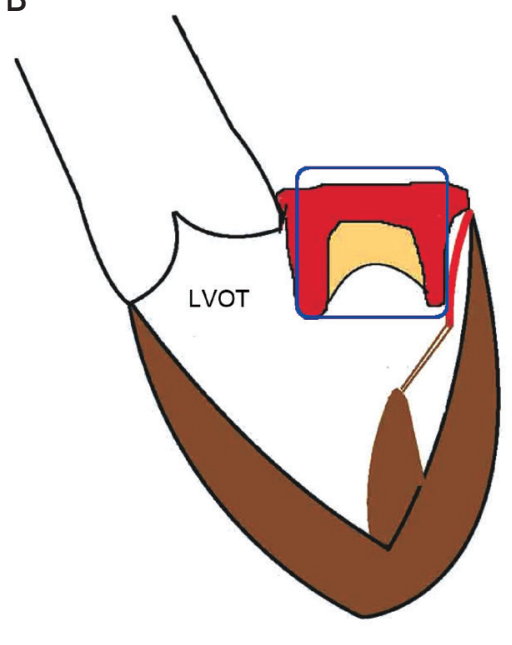

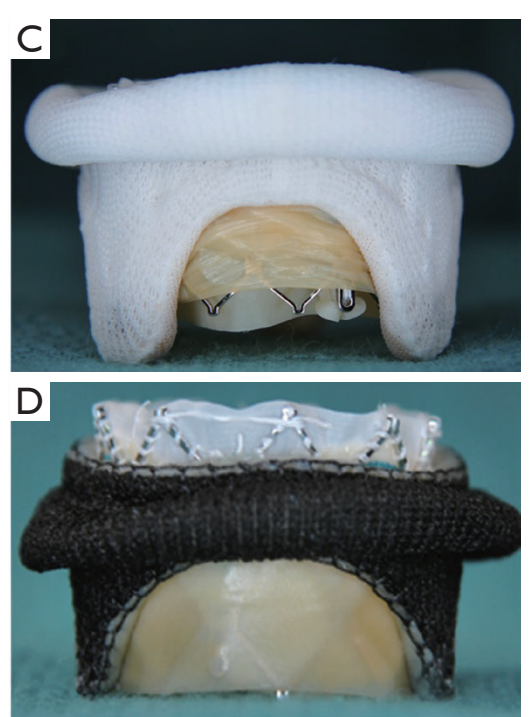

Figure 8 Effect of VIV and VIR on left ventricular outflow tract (LVOT). (A) Following VIR, the anterior mitral leaflet covers the THV and is pushed towards LVOT. (B) Following VIV, the surgical bioprosthetic leaflets cover the THV and can cause varying degrees of obstruction to LVOT. (C) Porcine mitral valve leaflet covering the SAPIEN valve. (D) Pericardial mitral leaflets covering the SAPIEN valve. The pericardial leaflets tend to be taller and tend to cover the THV more than the porcine counterparts. VIV, valve-in-valve; VIR, valve-inring; THV, transcatheter heart valve.

access site, fluoroscopic view for deployment is obtained. This step is critical, as the majority of the mitral procedures require a steep RAO view. In the fluoroscopic view, one has to confirm that radio-opaque objects such as ECG leads, wires, or other objects are not overlapping the field of interest. This can complicate the procedure if detected later. The view also allows the operators to understand their positions during the procedure. This is critical during a TA procedure as RAO views may interfere with the ability of the TA operator to stand close to the patient. Once this is confirmed, the patient is prepped for the surgery and draped. It is our practice to prepare the surgical site from neck to groin so as to provide access for TA route, TS route and median sternotomy if needed.

\section{Operation}

The TS approach is described in detail using a SAPIEN 3 valve, as it is the most common approach used today. Important steps for TA approach will be described separately.

\section{Access site preparation}

TS approach is performed through right or left femoral venous access. Right is preferred over the left as it allows a straighter trajectory for septal puncture. An additional venous access is secured through the left femoral vein with an 8-Fr sheath to insert a transvenous pacing wire, which is positioned in the right ventricle. Care is taken during the placement of the pacing wire. The threshold is checked and the pacing wire is secured with a stitch. A 5-Fr arterial line is secured if needed.

\section{Deployment view}

An ideal deployment view is obtained for easier positioning of the THV. Examples of ideal deployment view for bioprosthetic valve and ring is shown in Figure 12. Some valves and rings are difficult to visualize, and it is important that the plane is identified clearly during this process.

\section{Trans-septal puncture, septal dilatation, and valve crossing (Video 1)}

The site of the TS puncture is critical. Unlike some other mitral valve procedures, an ideal puncture for MVIV and MVIR is posterior-inferior (Figure 13). The puncture site should also be at least $3.5 \mathrm{~cm}$ from the mitral valve annulus. Puncture is performed under $\mathrm{x}$-plane using TEE guidance, which also ensures that there is no inadvertent injury to aorta or cardiac perforation.

There are various kits available for TS puncture. The 


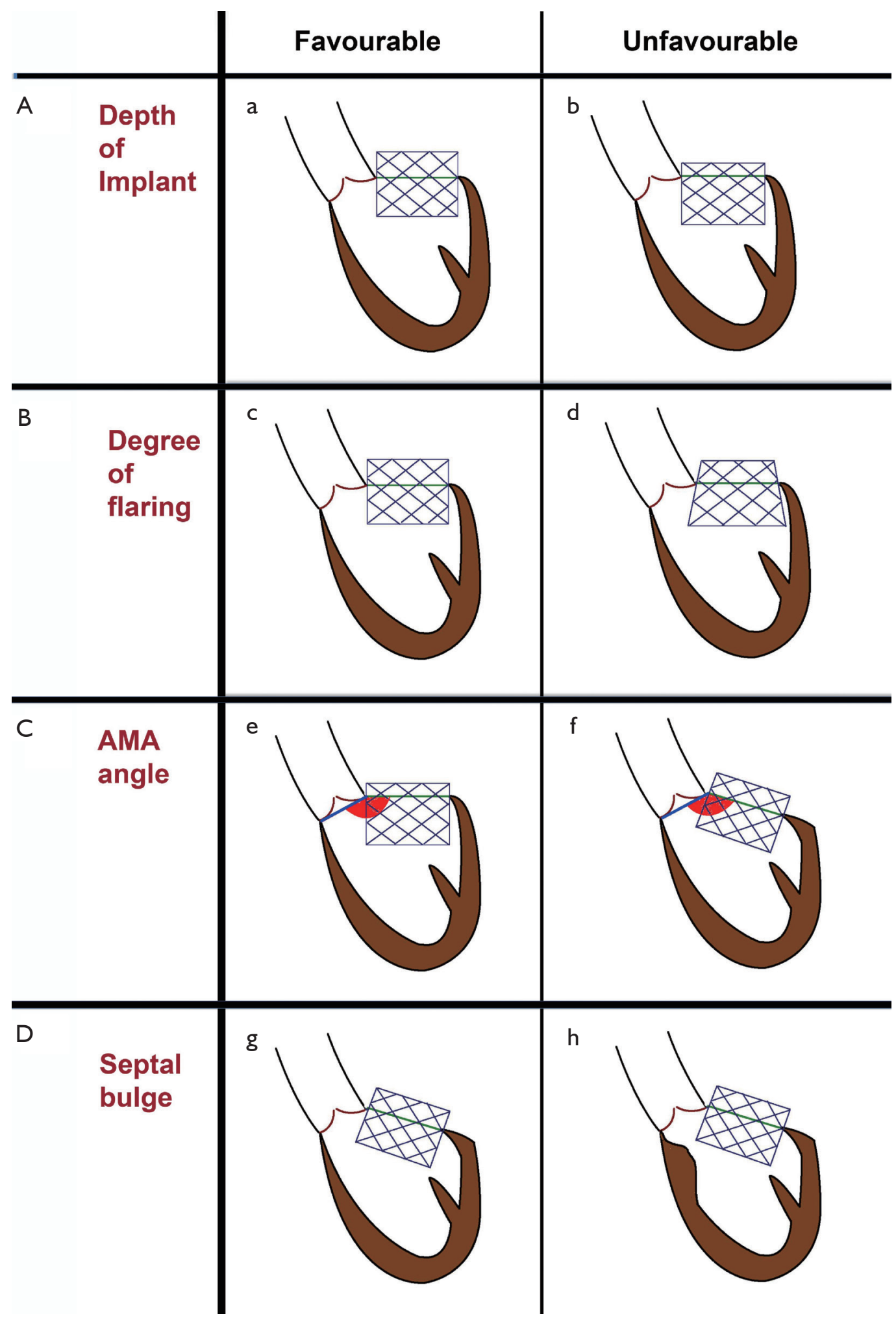

Figure 9 Factors influencing LVOTO after mitral VIV and VIR. (A) Depth of implant: the deeper the THV, the higher the LVOTO (b > a); (B) Flare in the left ventricle: increase in flare results in higher LVOTO (d > c); (C) Aorto-mitral annular angle: the less obtuse the angle, the higher risk of LVOTO (f > e); (D) Septal bulge: large septal bulge increases the risk of LVOTO (h > g). 
A

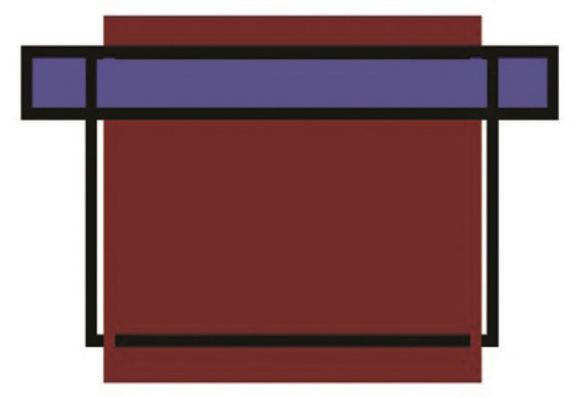

C

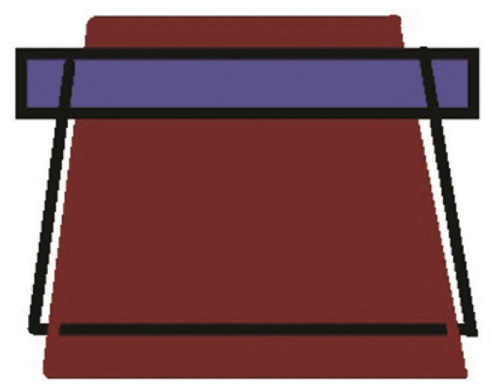

B

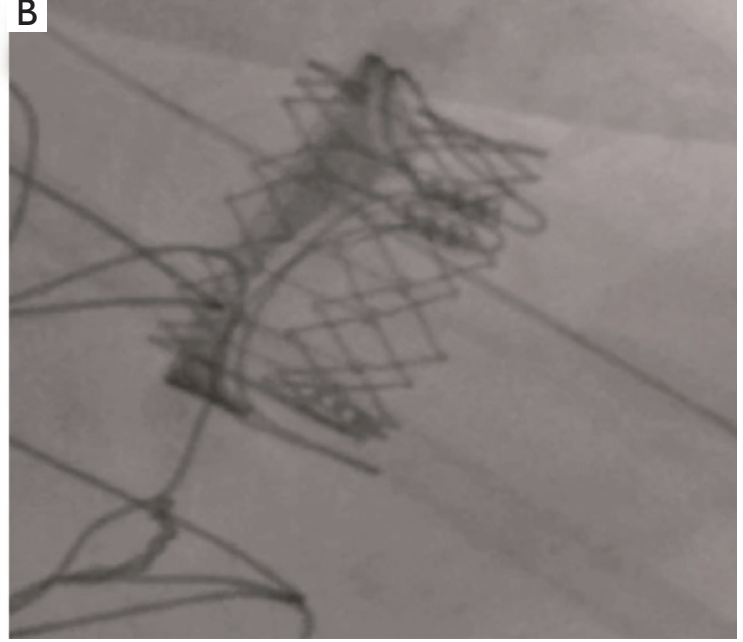

D

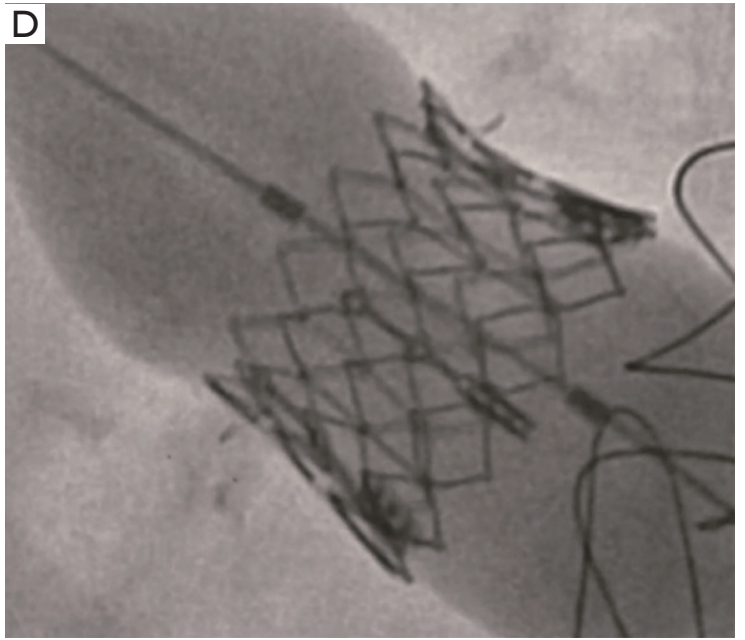

Figure 10 Risk of transcatheter heart valve embolization following mitral VIV. (A,B) Risk of embolization is high after parallel deployment; (C,D) risk of embolization is low or none after conical deployment. VIV, valve-in-valve.

commercially available Bayliss set is commonly used. Once the puncture is obtained, a guidewire is placed within the left atrium. Over the guidewire, a steerable guide catheter is placed and bent towards the mitral valve. This combination of various catheters and guidewires allows the operator to cross the mitral valve. The catheter is then advanced into the left ventricle, and a stiff wire (can be pre-shaped) is placed to provide support to deliver the THV. Various wires commonly used according to operator preference are Confida, Safari, Amplatz extra-stiff and Lunderquist. The septum is now dilated with 12 or 14 Fr Balloon to facilitate passage of the delivery system. Once the balloon is full, it should be flossed over to confirm adequacy of septostomy.
Once confirmed, balloon and steerable catheter are removed, and the E-sheath is inserted.

\section{Valve placement (Video 1)}

The Commander system is used for the TS approach. The most important part of this procedure is to check "correct orientation" of the SAPIEN 3 device. It is the exact reverse of the trans-femoral approach for native aortic valve (Figure 14). The delivery system is inserted with manufacturer logo oriented downwards, which ensures the direction of the flexion of the delivery system is towards the mitral valve (again exactly opposite of the TAVI procedure). The valve is pushed out of the sheath, aligned on the catheter, and 

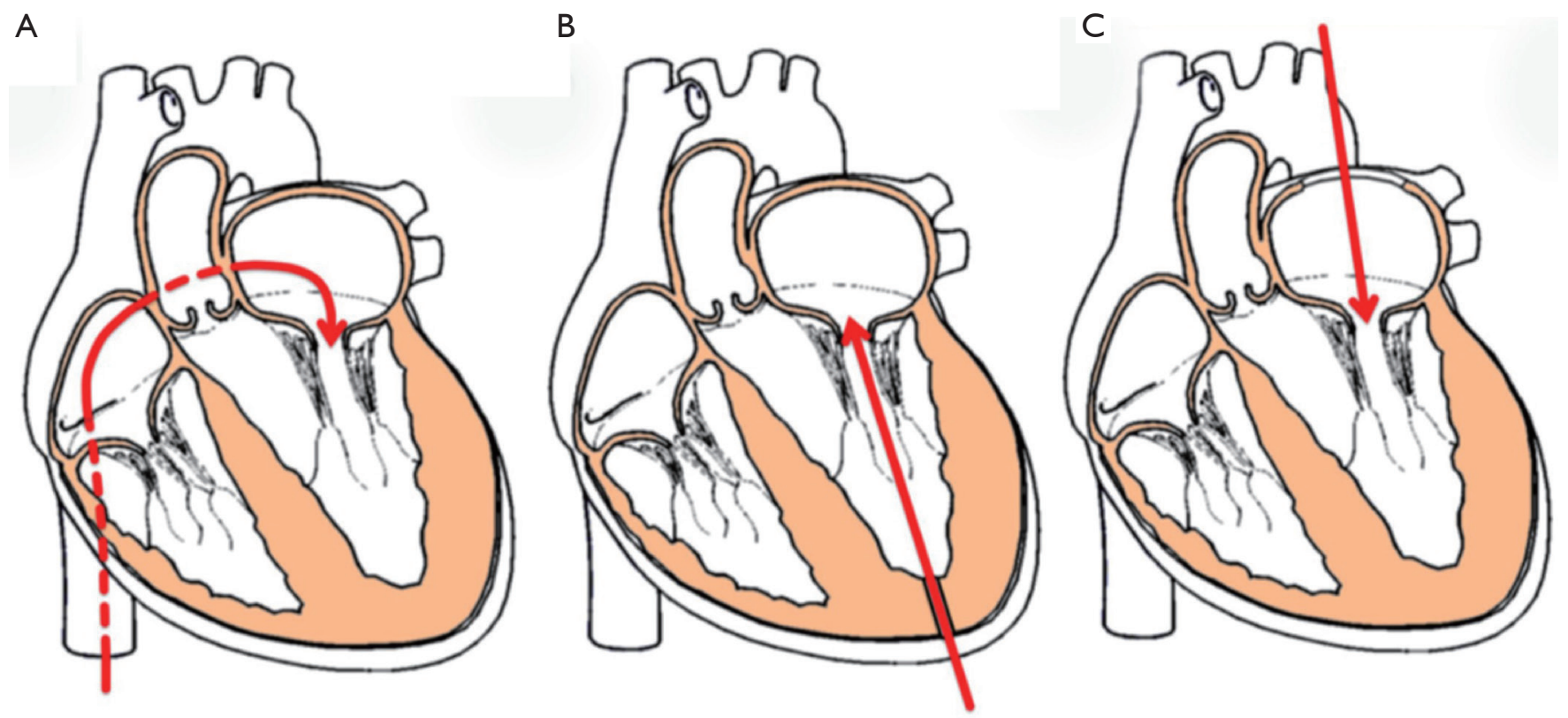

Figure 11 Access routes for mitral VIV and VIR. (A) Trans-septal approach: least invasive and most common; (B) trans-apical approach: more direct and allows for better control; (C) trans-atrial approach: used during open surgical implants. VIV, valve-in-valve; VIR, valve-inring.

then advanced through the septum into the left side of the heart. Once across, the flex catheter is retracted to expose the proximal balloon. Care is taken to ensure that the flex catheter still remains on the left side if possible. The valve is advanced through the bioprosthesis. The wire is managed so that it does not buckle or cause injury to the left ventricle. The valve is positioned as described earlier. TEE can be used to confirm ideal position. THV can be at an angle during initial positioning, but as the balloon inflates, THV tends to self-center.

\section{Valve deployment (Video 1)}

The final valvular deployment was assessed and considered appropriate, with 3-4 $\mathrm{mm}$ of the transcatheter prosthesis protruding into the left atrium for adequate stability and optimal sealing.

Valve deployment is performed under rapid pacing. Pacing at a rate of $180 /$ minute eliminates ejection and allows stable deployment. We like to perform a 2 -stage inflation, as it allows fine tuning with respect to ideal deployment. The first stage is inflation up to $10 \%$ to $15 \%$ followed by second stage to full deployment.

\section{Completion}

Once the valve is deployed, the delivery system is withdrawn partially. Valve function is assessed with TEE. Rarely, we acquire a left ventricular angiogram. If there is a need, postdilatation is performed with same or additional volume. The wire is then removed along with the delivery system. Shunt across the septum is assessed by TEE. If there is residual bidirectional or right to left shunt, the septostomy is closed with an atrial septal defect closure device. The E-sheath is removed, and the venous access site is closed with a single Perclose.

\section{Trans-apical approach}

It is similar to the TA approach used for TAVR. Once the left ventricular purse-string is placed, a guidewire is placed across the mitral valve into the left atrium. We like to use a softer wire to start with. Then, a catheter is placed into the left atrium, and the softer wire is exchanged for a stiff wire, similar to the TS approach. The Certitude system is used for TA approach. The Certitude sheath is inserted up to the $4 \mathrm{~cm}$ marker and secured. In certain cases, especially MVIV with bioprosthetic stenosis and MVIR where crossing 

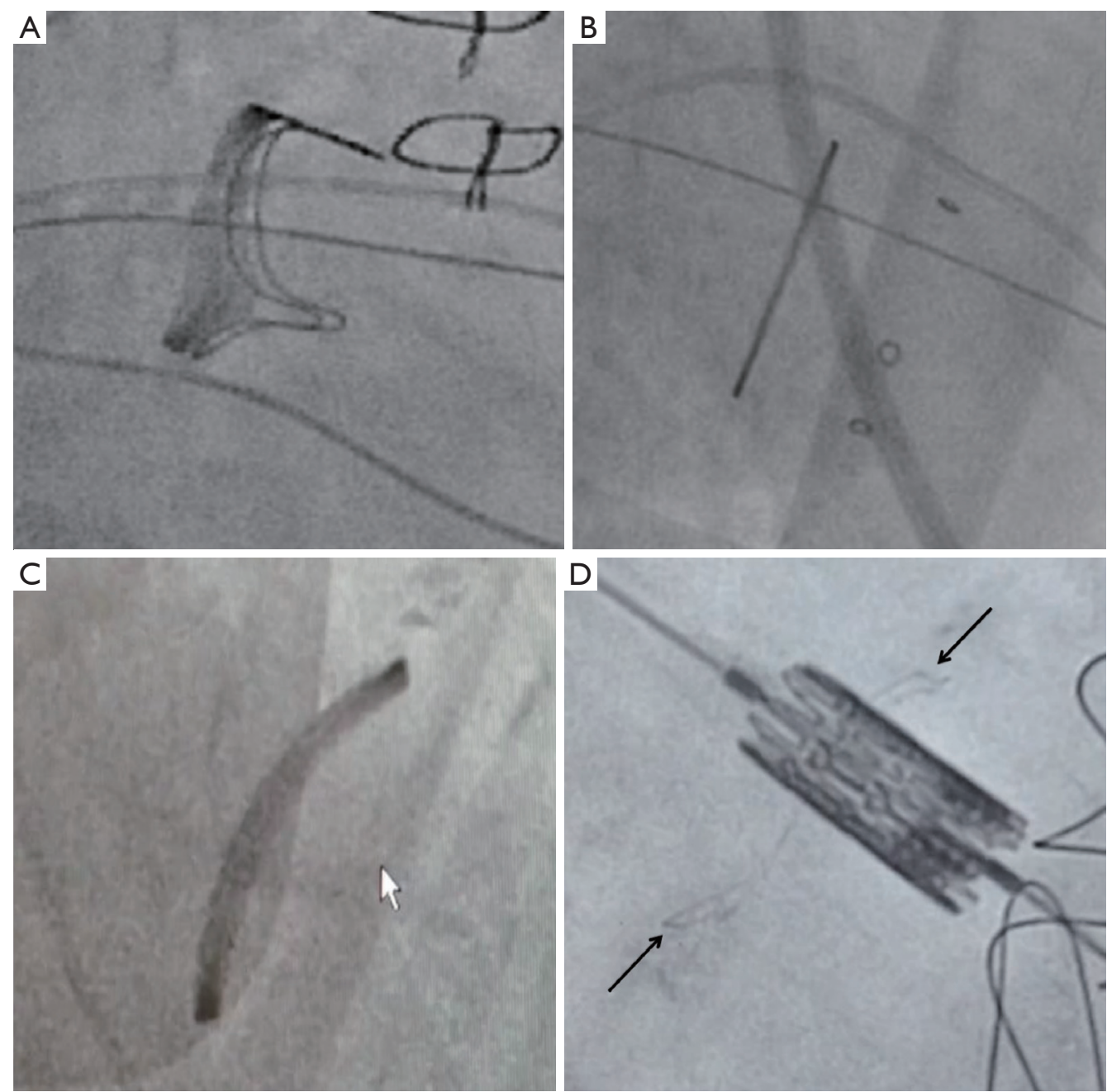

Figure 12 Ideal deployment view of fluoroscopy is important. Deployment views are shown with: (A) Perimount valve; (B) Hancock II valve; (C) St. Jude Rigid Saddle ring; (D) Epic/Biocor valve with SAPIEN XT positioned across it.
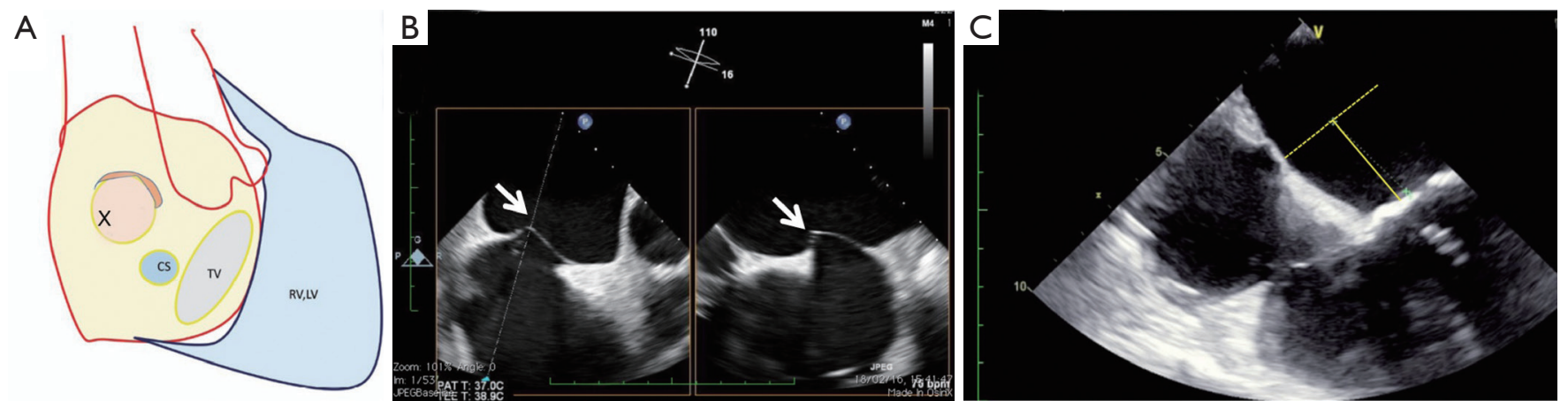

Figure 13 Trans-septal puncture for mitral VIV and VIR. (A) Schematic diagram showing fossa ovalis and the posterior-inferior site of septal puncture (X). (B) X-plane view of trans-septal puncture under TEE, demonstrating posterior-inferior puncture (white arrows). (C) Distance between the puncture and mitral annulus should be at least $3 \mathrm{~cm}$. 
A

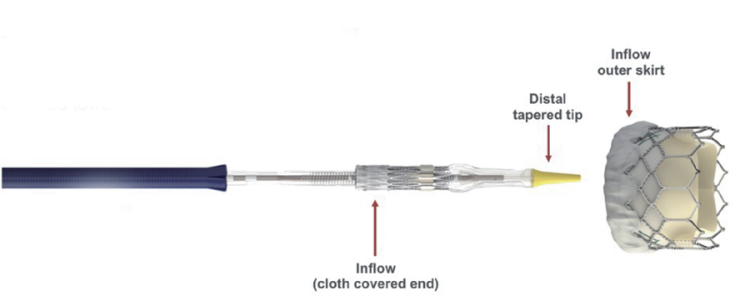

B

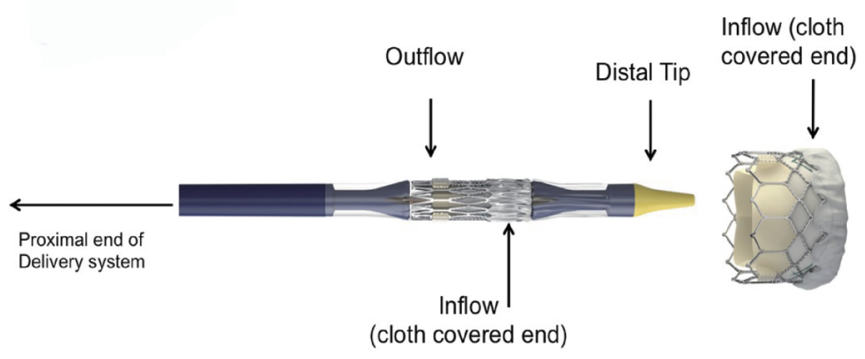

Figure 14 Orientation of the SAPIEN valve must be verified according to the approach. (A) For the Trans-septal approach using Commander system, the skirt is loaded proximally. (B) For the Trans-apical approach using Certitude system, the skirt is loaded distally.

valve is challenging due to stenosis, we like to introduce the sheath till the tip of the sheath is across the mitral annular plane. Then, the dilator is removed. SAPIEN Valve orientation is confirmed, and the delivery system is inserted. Once the SAPIEN valve is out of the sheath in the left atrium, the Certitude sheath is slowly backed out until reaching the $4 \mathrm{~cm}$ mark. The SAPIEN valve is now positioned and deployed, similar to the TS system. Post deployment checks are same. Once satisfied with the outcome, the sheath is withdrawn, often under rapid pacing, and purse-strings are tied. Hemostasis is achieved, and the chest is closed with a single chest drain.

\section{Comments}

\section{Clinical results}

The TMVR registry is a multicenter observational study that collected data on THV implantation in patients with failed mitral valve bioprosthesis, failed repair and MAC (16). A total of 521 patients were enrolled in a 9-year period (2009 until 2018), including 322 MVIV patients and 141 MVIR patients. The patient cohort was deemed high risk for surgery with a mean Society of Thoracic Surgeons (STS) score of $9 \%$ (16). A balloon expandable valve was utilized in $90 \%$ of the cases. In terms of procedural outcomes, conversion to surgery, need for a second valve implantation and LVOTO, MVIR fared worse compared to MVIV. Technical success was achieved in $94.4 \%$ of MVIV cases but only in $80.9 \%$ of MVIR. Post-procedural MR (> moderate) was observed more frequently in the MVIR group (18.4\% vs. $5.6 \%)$. All-cause thirty-day mortality was higher in the MVIR cohort than the MIV cohort (9.9\% vs. $6.2 \%)$. There were no significant differences found in terms of stroke, major bleeding, and acute kidney injury. The authors also demonstrated the effect of a learning curve on outcomes, as the latter half had lower rates of complications. Another important consideration involves valve thrombosis. This event occurred in 10 patients after MVIV, $50 \%$ of cases occurring within the first 3 months after the procedure, three between 3 months and 1 year, and two after 1 year. Overall, at 1 year, the incidence was higher in patients on antiplatelet therapy compared to patients on anticoagulation (60.6\% vs. $1.6 \%$; log-rank $\mathrm{P}=0.019)$, highlighting the importance of anticoagulation in this subset.

Mid-term and long-term outcomes of MVIV have also been reported by Dr. Ye and colleagues, who followed a cohort of thirty-one patients with failed mitral bioprosthetic valves who underwent VIV procedures with balloon expandable valves (17). The patients with degenerated mitral valve prosthesis had a STS score of $9.7 \%$ (high risk for redo-surgery). The study showed overall safety and feasibility of the transcatheter intervention, with a low rate of major adverse events and periprocedural complications. Thirty-day mortality and disabling stroke rates were $1.4 \%$, rate of life-threatening bleeding was $4.1 \%$, and no patient had more than mild paravalvular regurgitation. One mitral THV migrated aerially 2 months after the procedure, causing severe paravalvular leakage (PVL). Two patients on postoperative dual antiplatelet therapy developed valve thrombosis, which was resolved with the use of oral anticoagulants. Although there was just a trend in improvement of pulmonary pressures and left ventricular ejection fraction (LVEF) over the first 36 months, the authors described a significant improvement in heart failure symptoms and NYHA functional class up to 8 years. Estimated survival rates were $88.9 \%, 79.5 \%, 69.8 \%, 61.9 \%$, and $40.5 \%$ at $1,2,3,4$, and 5 years, respectively. 
Results of MVIR are not as promising as MVIV (16). Complications requiring conversion to surgery were threefold compared to VIV (2.8\% vs. $0.9 \%)$, the need for a second valve was as high as $12.1 \%$, and LVOTO occurred in $5 \%$ of the cases $(2.2 \%$ in VIV). Paravalvular regurgitation was common, as well as MR $\geq$ moderate (18.4\%). VIR showed higher mortality rates at thirty-days, greater major bleeding events and vascular complications, higher percentage of postprocedural acute kidney injury (AKI), and a lower procedural success (58.2\% vs. $73.6 \%$ for VIV).

Up to a few years ago, all cases of failed mitral valve prostheses were managed surgically with redo-surgery. The advent and development of transcatheter procedures gave the operators a valid alternative option, especially in high risk surgical patients. Kamioka and colleagues compared MVIV and redo surgery in a retrospective study, analyzing the 1-year echocardiographic and clinical outcomes. They demonstrated that there was no difference in 1-year mortality and thirty-days echocardiographic results, while mitral gradients were higher in the MVIV group (7.2 \pm 2.7 vs. $5.5 \pm 1.8 \mathrm{mmHg}$ ), although not clinically relevant (18). This means that good results can be achieved through a less invasive transcatheter intervention, avoiding high risk redo surgery. For future analysis, we need larger studies and should also assess mid-term and long-term results to better understand the real potential of this growing therapy area.

\section{Advantages of MVIR and MVIR}

MVIV or MVIR has following advantages: (I) avoidance of a redo-surgery; (II) shorter operation; (III) faster recovery; (IV) avoidance of blood transfusion.

As a result, MVIV and MVIR are slowly becoming first line therapy in high risk surgical patients, especially through the TS approach.

\section{Caveats}

This is indeed a promising new therapy, and most of what is known is derived from immediate- or short-term results only. Furthermore, results of MVIV have been much better than that of MVIR, owing to the design differences between various rings. This is an area of educating the operators to choose the right patient for MVIR. In MVIV, there are early signs that using the SAPIEN 23 valve may not result in good results as early as 1 year.

Although the majority of patients are already on anticoagulation, it is now considered reasonable to anticoagulate these patients for at least 6 months. Reported incidence of valve dysfunction seems to be higher with MVIV than aortic VIV.

LVOTO and risk of embolization has to be assessed properly. Similarly, going through a checklist for suitability of a ring is also important. If there is any doubt regarding procedural results, the patient should be followed up regularly with interval echocardiography.

\section{Acknowledgments}

Funding: None.

\section{Footnote}

Conflicts of Interest: The authors have no conflicts of interest to declare.

Open Access Statement: This is an Open Access article distributed in accordance with the Creative Commons Attribution-NonCommercial-NoDerivs 4.0 International License (CC BY-NC-ND 4.0), which permits the noncommercial replication and distribution of the article with the strict proviso that no changes or edits are made and the original work is properly cited (including links to both the formal publication through the relevant DOI and the license). See: https://creativecommons.org/licenses/by-nc-nd/4.0/.

\section{References}

1. Nishimura RA, Otto CM, Bonow RO, et al. 2017 AHA/ ACC Guideline for the management of patients with valvular heart disease: a report of the American College of Cardiology/American Heart Association Task Force on Pratice Guidelines. Circulation 2014;129:e521-643.

2. Badhwar V, Rankin JS, He X, et al. The Society of Thoracic Surgeons Mitral Repair/Replacement Composite Score: A report of the Society of Thoracic Surgeons Quality Measurement Task Force. Ann Thorac Surg 2016;101:2265-71.

3. Vongpatanasin W, Hillis LD, Lange RA, et al. Prosthetic heart valves. N Engl J Med 1996:335:407-16.

4. Bakaeen FG, Shroyer AL, Zenati MA, et al. Mitral valve surgery in the US Veterans Administration health system: 10-year outcomes and trends. J Thorac Cardiovasc Surg 2018;155:105-17.

5. Bapat V, Attia R, Redwood S, et al. use of transcatheter heart valves for a valve-in-valve implantation in patients 
with degenerated aortic bioprosthesis: technical considerations and results. J Thorac Cardiovasc Surg 2012;144:1372-9.

6. de Weger A, Ewe SH, Delgado V, et al. First-inman implantation of a trans-catheter aortic valve in a mitral annuloplasty ring: novel treatment modality for failed mitral valve repair. Eur J Cardiothorac Surg 2011;39:1054-6.

7. Bapat V, Mydin I, Chadalavada S, et al. A guide to fluoroscopic identification and design of bioprosthetic valves: a reference for valve-in-valve procedure. Catheter Cardiovasc Interv 2013;81:853-61.

8. Bapat VN, Attia R, Thomas M. Effect of valve design on the stent internal diameter of a bioprosthetic valve: a concept of true internal diameter and its implications for the valve-in-valve procedure. JACC Cardiovasc Interv 2014;7:115-27.

9. Bapat V. Valve-in-valve apps: why and how they were developed and how to use them. Eurointervention 2014;10:U44-51.

10. Conradi L, Silaschi M, Seiffert M, et al. Transcatheter valve-in-valve therapy using 6 different devices in 4 anatomic positions: clinical outcomes and technical considerations. J Thorac Cardiovasc Surg 2015;150:155765, 1567.e1-3; discussion 1565-7.

11. Bapat VN, Attia RQ, Condemi F, et al. Fluoroscopic guide to an ideal implant position for Sapien XT and CoreValve during a valve-in-valve procedure. JACC Cardiovasc Interv 2013;6:1186-94.

Cite this article as: Pirelli L, Hong E, Steffen R, Vahl TP, Kodali SK, Bapat V. Mitral valve-in-valve and valve-in-ring: tips, tricks, and outcomes. Ann Cardiothorac Surg 2021;10(1): 96-112. doi: 10.21037/acs-2019-mv-169
12. Bapat V, Pirone F, Kapetanakis S, et al. Factors influencing left ventricular outflow tract obstruction following a mitral valve-in-valve or valve-in-ring procedure, part 1 . Catheter Cardiovasc Interv 2015;86:747-60.

13. Blanke P, Naoum C, Dvir D, et al. Predicting LVOT obstruction in transcatheter mitral valve implantation: concept of the neo-LVOT. JACC Cardiovasc Imaging 2017;10:482-5.

14. Babaliaros VC, Greenbaum AB, Khan JM, et al. Intentional percutaneous laceration of the anterior mitral leaflet to prevent outflow obstruction during transcatheter mitral valve replacement: first-in-human experience. JACC Cardiovasc Interv 2017;10:798-809.

15. Bapat VV, Khaliel F, Ihleberg L. Delayed migration of Sapien valve following a trancatheter mitral valve-in-valve implantation. Catheter Cardiovasc Interv 2014;83:E150-4.

16. Yoon SH, Whisenant BK, Bleiziffer S, et al. Outcomes of transcatheter mitral valve replacement for degenerated bioprostheses, failed annuloplasty rings, and mitral annular calcification. Eur Heart J 2019;40:441-51.

17. Ye J, Cheung A, Yamashita M, et al. Transcatheter aortic and mitral valve-in-valve implantation for failed surgical bioprosthetic valves. An 8-year single-center experience. JACC Cardiovasc Interv 2015;8:1735-44.

18. Kamioka N, Babaliaros V, Morse MA, et al. Comparison of clinical and echocardiographic outcomes after surgical redo mitral valve replacement and transcatheter mitral valve-invalve therapy. JACC Cardiovasc Interv 2018;11:1131-8. 